\title{
Prevention of panic attacks and panic disorder in COPD
}

\author{
N. Livermore*,\#, L. Sharpe ${ }^{\#}$ and D. McKenzie ${ }^{\uparrow,+}$
}

ABSTRACT: This study examined whether cognitive behavioural therapy (CBT) could prevent the development or worsening of panic-spectrum psychopathology and anxiety symptoms in chronic obstructive pulmonary disease (COPD).

41 patients with COPD, who had undergone pulmonary rehabilitation, were randomised to either a four-session CBT intervention condition $(n=21)$ or a routine care condition $(n=20)$. Assessments were at baseline, post-intervention, and at 6-, 12- and 18-month follow-ups. Primary outcomes were the rates of panic attacks, panic disorder and anxiety symptoms. Secondary outcomes were depressive symptoms, catastrophic cognitions about breathing difficulties, disease-specific quality of life and hospital admission rates.

There were no significant differences between the groups on outcome measures at baseline. By the 18-month follow-up assessment, 12 (60\%) routine care group participants had experienced at least one panic attack in the previous 6 months, with two $(17 \%)$ of these being diagnosed with panic disorder, while no CBT group participants experienced any panic attacks during the followup phase. There were also significant reductions in anxiety symptoms and catastrophic cognitions in the СВТ group at all three follow-ups and a lower number of hospital admissions between the 6- and 12-month follow-ups.

The study provides evidence that a brief, specifically targeted CBT intervention can treat panic attacks in COPD patients and prevent the development and worsening of panic-spectrum psychopathology and anxiety symptoms.

KEYWORDS: Anxiety, chronic obstructive pulmonary disease, chronic obstructive pulmonary disease clinical investigations, panic attacks

ymptoms of anxiety are common in chronic obstructive pulmonary disease (COPD) and predict worse quality of life and functional level [1]. Many patients with COPD experience panic attacks, which are episodes of intense anxiety accompanied by symptoms of physical arousal [2]. To be diagnosed with panic disorder, patients must experience recurrent panic attacks, anticipatory anxiety about future attacks and some unexpected and unpredictable attacks (not always in situations inevitably causing dyspnoea in COPD) [2]. The prevalence of panic disorder in COPD has been estimated as up to 10-times greater than the population prevalence of $1.5-3.5 \%$ [2, 3].

This high prevalence of panic attacks and panic disorder in COPD is predicted by the cognitive model of panic anxiety [4]. In this model, panic attacks occur in healthy individuals when ambiguous physical sensations like shortness of breath are interpreted in a catastrophic way, such as indicating imminent asphyxia or death. Signs and symptoms of increased physical arousal follow, leading to an escalating cycle which results in panic. In COPD, worsening dyspnoea during physical activity is the defining symptom of an eventually fatal illness. It is easy to understand how vulnerable individuals with COPD, even early in the disease's course, may overestimate the immediate threat exertional dyspnoea presents and enter the cycle of panic. Anxious and depressive symptoms have been found to worsen over time in COPD patients, and this psychological deterioration has been only weakly correlated with decline in lung function [5]. In addition to affecting quality of life and functional level, anxiety and depression in COPD lead to increased reporting of medical symptoms, increased use of health services and up to double the health-related costs of patients with COPD alone [6]. Hence, treating anxiety in COPD patients, and so preventing panic disorder, is likely to improve patients' level of functioning and decrease costs. Cognitive behaviour therapy (CBT) is the treatment of choice for panic disorder in physically healthy adults [7, 8]. Evidence is increasing that CBT produces its effects on panic
AFFILIATIONS

${ }^{*}$ Dept of Liaison Psychiatry, Prince of Wales Hospital,

'Dept of Respiratory Medicine, Prince of Wales Hospital,

\# School of Psychology, University of Sydney, and

+Faculty of Medicine, University of New South Wales, Sydney, NSW, Australia.

CORRESPONDENCE

N. Livermore

Dept of Liaison Psychiatry

Prince of Wales Hospital

Barker St

Randwick 2034

NSW

Australia

E-mail: Nicole.Livermore@

sesiahs.health.nsw.gov.au

Received:

April 102009

Accepted after revision:

Aug 272009

First published online:

Sept 092009

European Respiratory Journal Print ISSN 0903-1936 Online ISSN 1399-3003 
attacks and panic disorder by decreasing catastrophic beliefs about ambiguous physical sensations $[9,10]$.

The results of several controlled trials using self-report questionnaires have indicated that CBT can decrease anxiety symptoms in patients with COPD; however, the effects of CBT on panic attacks and the development of panic disorder in COPD have not been assessed [11-15]. Intervening early with individuals at risk of panic disorder can prevent the personal and economic effects of the disorder becoming entrenched [16]. In the current study, we therefore evaluated a brief CBT intervention specifically directed at panic-related anxiety in COPD. The hypothesis was that CBT would be more effective than routine care, which included pulmonary rehabilitation, in preventing the development and/or worsening of panicspectrum psychopathology and anxiety symptoms in patients with COPD, over an 18-month period.

\section{METHODS}

\section{Study subjects}

The study was conducted at a teaching hospital in Sydney, Australia. The trial was approved by the University and Hospital Ethics Committees (Sydney, Australia), and all subjects gave written consent. 96 consecutive COPD patients who met the study's inclusion criteria were identified in the respiratory medicine department's outpatient clinics or inpatient ward and invited to participate. 52 (54\%) of these patients volunteered. This recruitment rate was similar to the $55 \%$ reported by KUNIK et al. [17]. Baseline assessments were delayed until $\geqslant 1$ month after any hospital admission for an infective exacerbation of COPD. All subjects had a diagnosis of moderate to severe COPD (forced expiratory volume in $1 \mathrm{~s}$ (FEV1) post-bronchodilator $<60 \%$ predicted and FEV1/forced vital capacity $(\mathrm{FVC})<0.7)$, corresponding to the Global Initiative for Chronic Obstructive Lung Disease stages II or III [18]. They were receiving ongoing outpatient treatment in the respiratory medicine department, where they had also attended an integrated, comprehensive outpatient pulmonary rehabilitation programme. The possible confounding factor of pulmonary rehabilitation attendance, which has been associated with short-term reductions in questionnaire-measured symptoms of anxiety and depression, was thus controlled for [19]. All subjects were being treated with a combination longacting $\beta_{2}$ agonist/inhaled corticosteroid and a long-acting anticholinergic. None was on high dose oral corticosteroids. Exclusion criteria were lack of English literacy (due to the requirement for completion of standardised questionnaires unavailable in other languages) and the presence of any other serious physical illness or psychiatric disorder, including panic disorder. Individuals with panic disorder were excluded because of the study's preventative focus and because an extended psychological intervention was considered necessary for such patients [20]. At initial assessment, the Panic Disorder and Agoraphobia sections of the Anxiety Disorders Interview Schedule of the Diagnostic and Statistical Manual for Mental Disorders, 4th Edition, (ADIS-IV) were administered, along with relevant questionnaires [21-24]. 11 individuals were then found ineligible for the study: nine $(17 \%)$ were diagnosed with panic disorder and two (4\%) were diagnosed with major depression. The prevalence of panic attacks $(19 \%, n=10$ out of
52) and panic disorder ( $17 \%, \mathrm{n}=9$ out of 52) at initial assessment were within the ranges expected [3].

\section{Procedure}

Assignment

We used the Excel Bernoulli Function to generate a random sequence of numbers, with a $50 \%$ chance of each of the two groups occurring. Randomisation was linked to subject numbers and was concealed until after the baseline assessment was completed. 21 participants were randomised to the CBT group and 20 to the routine care group (fig. 1).

\section{Assessments}

Measures were administered at baseline, post intervention (56 weeks later) and at 6-, 12- and 18-month follow-up assessments. Pulmonary function tests (FEV1 and FVC) were carried out at baseline by trained laboratory scientists.

\section{CBT intervention}

The standardised CBT intervention comprised four, individually administered, 1-h sessions. These weekly outpatient sessions commenced within 2 weeks after baseline assessment. The patient manual for the intervention was developed by, and is available from, N. Livermore, an experienced clinical psychologist who also administered the intervention. The manual included strategies effective for the prevention and treatment of panic disorder in younger adults [25]. Adaptations were required for an elderly population with COPD [20, 26-28] Components of the intervention therefore included psychoeducation about CBT, the effects of the stress response on breathing

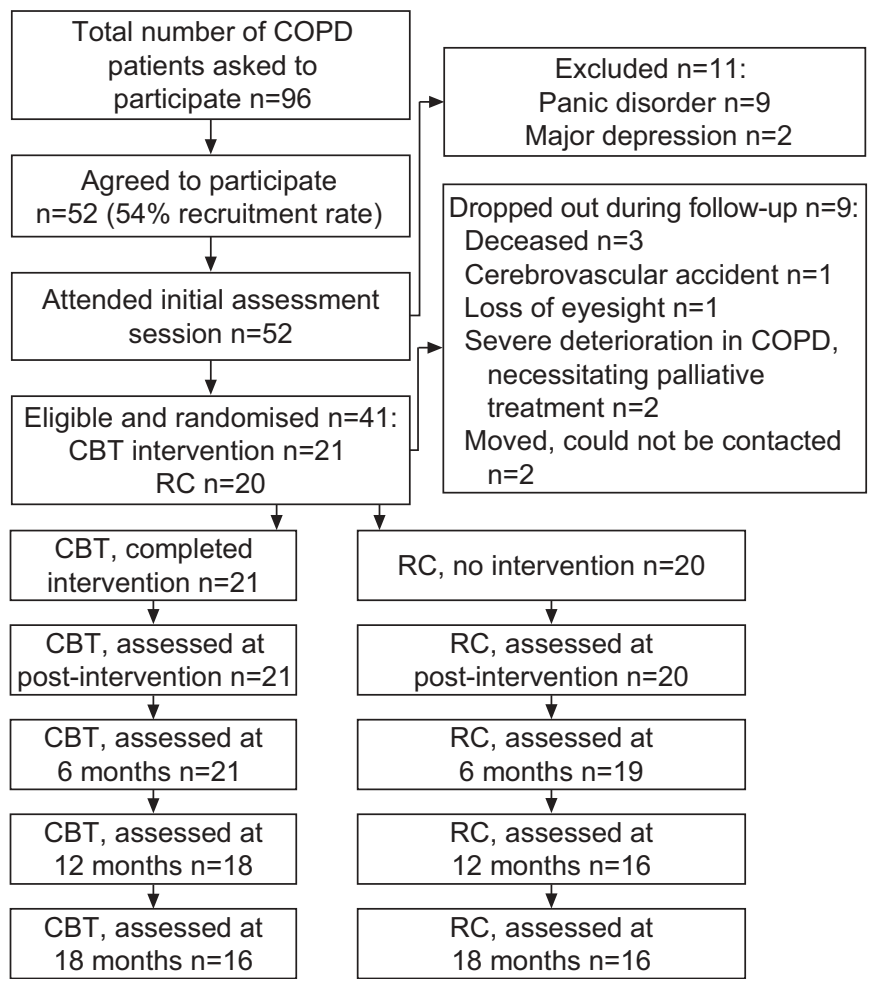

FIGURE 1. Numbers/attrition of participants from enrolment to 18-month follow-up. COPD: chronic obstructive pulmonary disease; CBT: cognitive behaviour therapy; $\mathrm{RC}$ : routine care 
and the cycle of panic anxiety in COPD; training in cognitive challenging of unhelpful cognitions; training in "pursed lip breathing"; reinforcement of activity planning and "pacing"; the development of a personalised "good coping plan"; and problem-solving to address any barriers to good coping.

\section{Measures}

Demographics

Age, education, occupation before retirement, years since diagnosis of COPD, cigarette smoking history, body mass index, number of days per week when alcohol was consumed, number of alcoholic drinks consumed on these days, incidence of home $\mathrm{O}_{2}$ use, FEV1 post-bronchodilator, FVC post-bronchodilator and the FEV1/FVC ratio post-bronchodilator were recorded.

Measures for assessing primary and secondary outcome variables The study's primary outcome variables were rates of panic attacks and panic disorder, and anxiety symptomatology. Panic attacks and panic disorder were diagnosed with the ADIS-IV, the gold standard clinical interview for diagnosing anxiety disorders [21]. Although panic disorder is more difficult to diagnose when comorbid with respiratory disease, it is identifiable, as mentioned above, when patients have anticipatory anxiety about future panic attacks and experience some unpredictable attacks not solely precipitated by unavoidable triggers for dyspnoea in COPD (such as physical exertion or infective exacerbations of the disease) [2]. N. Livermore carried out the assessments and intervention, so ADIS-IV interviews were recorded to decrease any possible influence of experimenter bias. Inter-rater reliability was assessed by having another clinical psychologist, blind to treatment condition, listen to a randomly selected $20 \%(n=40)$ of all recordings $(n=205)$. Diagnostic agreement between the two raters for these interviews was $100 \%$. The other primary outcome variable, anxiety symptomatology, was assessed using the anxiety subscale of the Hospital Anxiety Depression Scale (HADS), a 14-item questionnaire with good validity and reliability [22].

Four secondary outcome variables were investigated. Depressive symptoms were measured using the depression subscale of the HADS [22]. The Interpretation of Breathing Problems Questionnaire (IPBQ) was included as a measure of illness-specific catastrophic cognitions in patients with COPD [23]. The St. George's Respiratory Questionnaire (SGRQ) was used to assess quality of life [24]. Data on the number of COPD-related admissions for each participant were obtained from the hospital's database.

\section{Sample size}

As this was the first study to investigate the efficacy of a specific CBT intervention in preventing panic-spectrum psychopathology in COPD, it was difficult to determine the effect size to use as a basis for predicting power. Power calculations, based on between-group differences in anxiety scores at post intervention (effect size $=0.53$ ) in a previous CBT study, indicated that a sample of 52 (26 in each group) would allow detection of changes at $5 \%$ significance with a power of over $95 \%$ for anxiety [14]. However, the study from which this effect size was drawn used a generic CBT programme. We expected larger effect sizes for the primary outcome variables (rates of panic attacks/panic disorder and anxiety symptomatology as measured by the HADS) from a more directly focused CBT approach for panic-spectrum psychopathology, anticipating that our total sample size of 41 would provide sufficient power to detect differences between groups on these variables.

\section{Statistical analysis}

SPSS 12.0 (SPSS, Chicago, IL, USA) was used for all analyses. Baseline differences between the groups were investigated using one-way ANOVA. It was planned to include any variable found to differ significantly between the groups as a covariate. A series of mixed model 2 (group: CBT versus routine care) $\times 2$ (time: pre- versus post-intervention/followup) ANCOVAs were conducted. Only interaction effects, testing differences in changes in the two groups' results over time, are reported. Chi-squared tests were used to compare the incidence of panic attacks at each assessment. The MannWhitney test was used to compare the number of hospital admissions, as these were not normally distributed. Completer and intention to treat analyses were carried out, with no substantive difference in the pattern of results. Results of the intention to treat analysis are reported here. Statistical significance was defined as a p-value $<0.05$.

\section{RESULTS}

\section{Baseline differences}

There were no significant differences at baseline assessment between CBT and routine care participants on the number of males and females, age, years since diagnosis, cigarette smoking status, body mass index, home $\mathrm{O}_{2}$ status, $\mathrm{FEV} 1$ post-bronchodilator, FVC post-bronchodilator or FEV1/FVC ratio post-bronchodilator (table 1). They did not differ on number of alcoholic drinks per day, but did differ on number of days per week on which some alcohol was consumed (table 1). We therefore controlled for the latter variable in subsequent analyses. All participants were Caucasian and retired (primarily from blue collar employment). Only one participant in each condition had completed high school, and one in each condition had English as a second language. There were no differences between the CBT and routine care groups at baseline on any of the study's outcome measures (table 1).

\section{Primary outcomes}

Although there were no differences between the groups on the ADIS-IV at baseline assessment $(p=0.48)$, there were significant differences post-intervention $(p=0.003)$ and at the 6 month ( $\mathrm{p}<0.001), 12$-month $(\mathrm{p}<0.001)$ and 18-month $(\mathrm{p}<0.001)$ follow-ups in the proportion who had experienced at least one panic attack since the previous assessment (fig. 2). Four (20\%) of the participants in the CBT and six $(30 \%)$ in the routine care group had experienced at least one panic attack in the month prior to baseline assessment. However, all CBT group participants were asymptomatic post-intervention, and remained so over the 18-month follow-up period. No further cases of panic attacks developed in the CBT group. In the routine care group, $35 \%$ of participants had experienced at least one panic attack by post-intervention and $60 \%$ by the 18 month follow-up. Two of the 12 routine care group participants, who had experienced at least one panic attack by the 18month follow-up, met criteria for panic disorder; one with and 


\begin{tabular}{|c|c|c|c|}
\hline & Intervention & $\begin{array}{l}\text { Routine } \\
\text { care }\end{array}$ & p-value \\
\hline Subjects $n$ & 21 & 20 & \\
\hline Female & $12(57)$ & $11(55)$ & 0.89 \\
\hline Age yrs & $73.2 \pm 6.4$ & $73.5 \pm 8.1$ & 0.91 \\
\hline Time since COPD diagnosis yrs & $6.5 \pm 4.9$ & $6.4 \pm 5.6$ & 0.96 \\
\hline Current smoking & $3(14)$ & $2(10)$ & 0.68 \\
\hline BMI $\mathbf{k g} \cdot \mathrm{m}^{-2}$ & $25.7 \pm 4.3$ & $26.2 \pm 5.3$ & 0.80 \\
\hline $\begin{array}{l}\text { Alcohol consumed each week } \\
\text { days }\end{array}$ & $2.5 \pm 1.5$ & $3.4 \pm 1.1$ & $0.04^{*}$ \\
\hline Standard drinks & $0.4 \pm 0.9$ & $0.2 \pm 0.4$ & 0.28 \\
\hline On home $\mathrm{O}_{2}$ therapy & $4(19)$ & $5(25)$ & 0.65 \\
\hline FEV 1 post-bronch $\%$ pred & $57.0 \pm 25.1$ & $51.1 \pm 15.0$ & 0.11 \\
\hline FVC post-bronch \% pred & $88.5 \pm 16.8$ & $80.3 \pm 12.9$ & 0.10 \\
\hline FEV1/FVC ratio post-bronch & $0.67 \pm 0.2$ & $0.62 \pm 0.17$ & 0.37 \\
\hline $\begin{array}{l}\text { COPD admissions in } 6 \text { months } \\
\text { prior to study }\end{array}$ & $13(62)$ & $14(70)$ & 0.60 \\
\hline $\begin{array}{l}\geqslant 1 \text { panic attack in previous } \\
\text { month }\end{array}$ & $4(20)$ & $6(30)$ & 0.48 \\
\hline HADS anxiety subscale score & $5.2 \pm 2.9$ & $5.9 \pm 2.7$ & 0.45 \\
\hline $\begin{array}{l}\text { HADS depression subscale } \\
\text { score }\end{array}$ & $3.9 \pm 2.1$ & $4.1 \pm 2.8$ & 0.55 \\
\hline IBPQ score & $7.0 \pm 4.3$ & $8.2 \pm 4.8$ & 0.44 \\
\hline SGRQ score & $40.3 \pm 15.1$ & $47.9 \pm 17.9$ & 0.15 \\
\hline
\end{tabular}

Data are presented as mean \pm SD or $n(\%)$, unless otherwise stated. COPD: chronic obstructive pulmonary disease; BMl: body mass index; FEV1: forced expiratory volume in $1 \mathrm{~s}$; post-bronch: post-bronchodilator; \% pred: \% predicted for age; FVC: forced vital capacity; HADS: Hospital Anxiety Depression Scale; IBPQ: Interpretation of Breathing Problems Questionnaire; SGRQ: St George's Respiratory Questionnaire. *: $p<0.05$ on ANOVA.

one without agoraphobia. This result represents an absolute risk reduction provided by the CBT intervention of $43 \%$, indicating that three patients needed to be treated to prevent one case of panic attacks developing during the 18-month follow-up period (95\% CI 1.5-5.9).

On the HADS anxiety subscale, there was no significant time by group interaction post-intervention $(p=0.22)$ (fig. 3a). However, there were significant time by group interactions at the 6-month $(\mathrm{p}=0.006), 12$-month $(\mathrm{p}=0.008)$ and 18-month $(\mathrm{p}=0.001)$ follow-ups, favouring the CBT group. At each follow-up, mean anxiety scores for the CBT group were in the nonclinical range, while mean scores for the routine care group were above the cut-off score $(>7)$ for possible clinically significant anxiety [22]. Only one (5\%) of the CBT participants, compared with nine $(45 \%)$ of the routine care participants had scores above this cut-off at the 18-month follow-up.

\section{Secondary outcomes}

There were significant time by group interactions on the IPBQ at the 6-month $(p=0.012), 12$-month $(p=0.001)$ and 18-month $(\mathrm{p}=0.002)$ follow-ups, again favouring the CBT group (fig. $3 b$ ). There were no significant interactions on the HADS depression subscale at any follow-up ( $p>0.11$ ) (fig. 3c). Similarly, there were no significant interactions on the SGRQ $(p>0.40)$. There

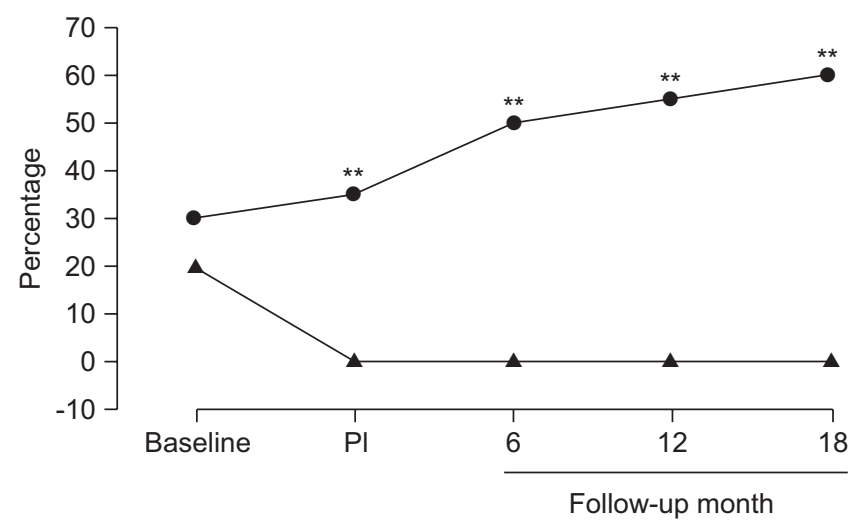

FIGURE 2. Percentage of subjects who had experienced at least one panic attack in the previous month (at baseline and post-intervention (PI)) or previous 6 months (at the 6-, 12- and 18-month follow-ups) in the cognitive behavioural therapy group $(\mathbf{\Lambda})$ and the routine care group $(\boldsymbol{\bullet})$. Differences between the groups were significant at post-intervention and all follow-ups. ${ }^{*}: p<0.01$.

was a significant difference in the number of hospital admissions for exacerbations of COPD between the 6- and 12-month follow-ups, with four admissions for the CBT and nine for the routine care group $(p=0.04)$. Differences in admission numbers at other time points (between postintervention and the 6-month follow-up, there were two for the CBT and seven for the routine care group, and between the 12- and the 18-month follow-ups, there were three for the CBT and five for the routine care group) were not significant $(\mathrm{p}>0.23)$.

\section{DISCUSSION}

Our findings support the hypothesis that CBT is more efficacious than routine care in preventing the development or worsening of panic-spectrum psychopathology in COPD patients. At the 18-month follow-up, 12 (60\%) of the routine care participants had experienced at least one panic attack in the previous 6 months, with two $(17 \%)$ of those meeting criteria for panic disorder. In contrast, no-one in the CBT group had experienced a panic attack during the intervention or follow-up period, even though at baseline, four patients had experienced at least one panic attack in the previous month. This result indicates that CBT had a treatment effect, as well as a preventative impact. The number needed to treat analysis provided further evidence of the intervention's usefulness, with approximately one case of panic attack prevented for every three patients treated over an 18-month follow-up period. There was also a decrease in anxiety symptoms, as measured by the HADS, over time in the CBT group, in contrast to a mean increase in symptoms to a level of possible clinical significance in the routine care group. Nearly half $(45 \%)$ of the routine care group had anxiety scores in either the clinical or possible clinical ranges at the 18-month follow-up. Further, the CBT group had significantly fewer COPD-related hospital admissions between the 6- and 12-month follow-ups. These results suggest that this brief CBT intervention had a specific impact on panic-related anxiety. The only treatment effect on secondary outcome questionnaires was found for the IPBQ, which assesses the degree to which individuals with COPD interpret potentially anxiety-provoking scenarios in a 

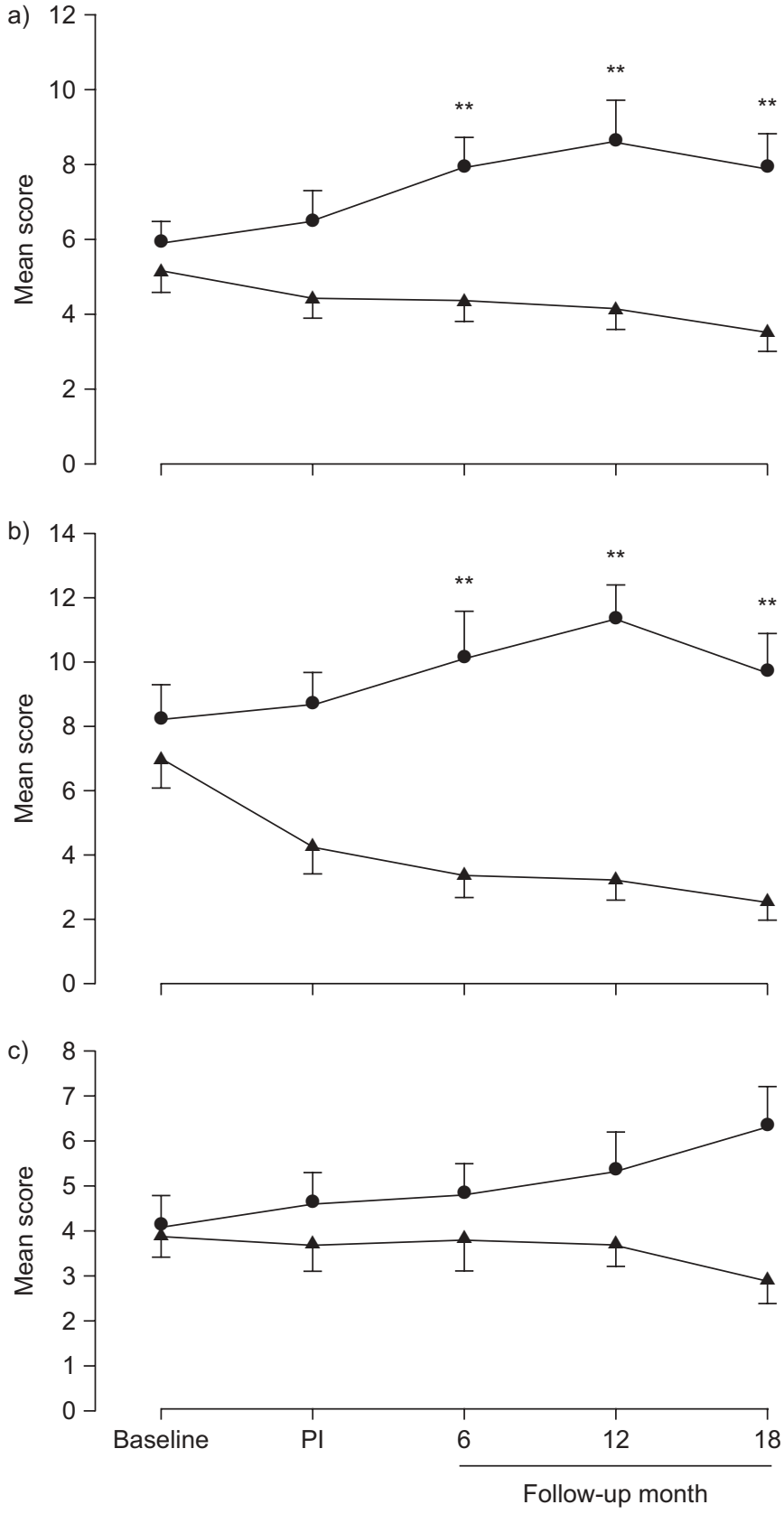

FIGURE 3. Mean scores at baseline, post-intervention (PI), and at 6-, 12- and 18-month follow-ups for the cognitive behavioural therapy group $(\mathbf{\Lambda})$ and the routine care group ( $\bullet$ on a) the Hospital Anxiety Depression Scale's anxiety subscale b) the Interpretation of Breathing Problems Questionnaire and c) the Hospital Anxiety Depression Scale's depression subscale. Data are presented as mean \pm SEM. Higher scores indicate worse symptomatology. ${ }^{* *}: p<0.01$.

catastrophic way. This outcome corresponds with the central roles of both the catastrophic interpretation of ambiguous physical sensations, such as dyspnoea, in the cognitive model of panic anxiety and the changes in catastrophic interpretations in mediating the effects of CBT for panic symptoms $[4,9,10,20]$.

Five previous controlled trials have provided evidence that CBT can decrease anxiety symptoms in COPD patients who have not been selected on the basis of existing psychopathology
[11-15]. However, in all five studies, anxiety symptoms were measured solely by self-report questionnaire and only one of the studies included a follow-up assessment (at 3 months) [12]. KUNIK et al. [17] recently conducted a randomised controlled trial of a group CBT intervention for selected COPD patients with moderate-to-severe anxiety and/or depressive symptoms, as measured by questionnaire. At baseline, 38\% of subjects were diagnosed with unspecified anxiety disorders; however, diagnostic status after treatment was not reported. Patients receiving CBT showed similar, clinically significant improvements, maintained over 10 months, to those receiving only COPD education: mean scores for both groups on the questionnaire measures of anxiety and depressive symptoms decreased from the "moderate" to the "mild" range. Reasons for the negative results of the study include the possibilities that the control intervention had an anxiety-reducing effect and that the CBT intervention was too general in content to fully address the symptoms of individuals with clinical anxiety and/or depression [17]. The current study is the first to focus on formally diagnosed panic-spectrum psychopathology in COPD. It is also the first psychological treatment study to include a follow-up period longer than 10 months [17].

Although the findings of the current study are encouraging, there are limitations to be noted. The first of these is sample size. With such a relatively small sample, there may be some concerns about statistical stability. The sample size may also limit extrapolation of the results to the entire population of patients with COPD. It is additionally possible that some of the null findings in secondary outcome variables were due to insufficient statistical power. However, effect sizes for secondary outcome questionnaires other than the IPBQ were small, with the largest (0.29) being observed for the HADS depression subscale. Any changes in these secondary outcome questionnaires are therefore unlikely to be clinically significant. The intervention studied here particularly targeted panic-spectrum psychopathology. For other psychological problems, such as depressive disorders, a different intervention would be appropriate. A further limitation of the study was that neither the assessor nor the participants were blind to treatment condition. To decrease any potential effects of experimenter bias, a fully structured diagnostic interview was administered. Further, we checked interrater reliability with a blind assessor on a $20 \%$ subsample of all interviews ( $n=40$ out of 205$)$, where $100 \%$ concordance was achieved. The consistency of results between self-report, diagnostic interview and hospital admission data also decreases the likelihood that experimenter bias could account for the results.

These limitations notwithstanding, there are several strengths of the study. This is the first trial with COPD patients to specifically address panic-spectrum psychopathology and the first to formally measure diagnostic status for panic attacks and panic disorder at all assessment points. Subject retention was excellent, with $100 \%$ of those randomised completing the post-intervention assessment and $78 \%$ completing the study. The CBT intervention was sufficiently brief to be offered to all patients who had attended pulmonary rehabilitation, but sufficiently long for relevant skills to be practised. The intervention responds to the recent call by BOURBEAU and VAN DER PALEN [29] for further programmes to help COPD patients self-manage their care as effectively as possible. The 
follow-up period was longer than in previous studies and the effects of the intervention were maintained. The trial provides the first evidence that untreated panic attacks in COPD do not spontaneously remit and are a risk factor for panic disorder [30]. POLLACK and SMOLLER [31] have proposed that when an anxiety disorder develops comorbidly with a respiratory disease (as in nearly $40 \%$ of the subject in KUNIK et al. [17]), treatment of the anxiety disorder may be more prolonged and difficult [20]. Interventions such as the current one may help to prevent the high costs of entrenched panic disorder for patients and for health services [6].

If we had provided CBT only to COPD patients with existing high anxiety, we would have missed some patients who went on to develop panic-spectrum psychopathology [5]. We therefore suggest that this type of brief, CBT self-management intervention should be routinely made available to all COPD patients, perhaps as a component of pulmonary rehabilitation programmes. Doing so could normalise contact with a mental health professional for elderly patients who may otherwise resist such contact [32]. Using a "stepped care" approach, it could prepare the ground for treatment of psychological disorders when necessary for some patients, while simultaneously decreasing the likelihood of panic-spectrum psychopathology developing in others [30, 33, 34].

A full cost-benefit analysis was beyond the scope of the study. However, hospitalisation is the major contributor to healthcare costs in COPD [35]. For the period between the 6- and 12month follow-ups, with an average admission length of 7 days at a cost to the health service of AU\$800 per day, the direct hospitalisation cost for the CBT group was approximately AU\$22,400 and the routine care group AU\$50,400. The total cost of the clinical psychologist's time with all 21 patients was AU $\$ 3,650$. We calculated an admission rate of 0.19 (four out of 21) for the CBT group and 0.45 (nine out of 20) for the routine care group for this 6-month period. For a pool of 500 patients (a typical case load for a large referral centre) over a 12-month period, the admission costs would be AU $\$ 1,064,000$ versus AU $\$ 2,520,000$, a difference of AU\$1,456,000 at a cost for the clinical psychologist's time of about AU\$87,000. The net saving of AU\$1,369,000 suggests that brief, preventative CBT interventions may decrease the burden of COPD not only to patients and carers but to healthcare systems, adding to any decrease achieved by integrated, comprehensive pulmonary rehabilitation [36].

In conclusion, the results of this study indicate that brief, specifically targeted CBT can treat panic attacks in COPD patients and prevent the development or worsening of panicspectrum psychopathology and anxiety symptoms. The intervention was also associated with a decrease in hospital admissions. Larger scale prevention trials are warranted by the current findings, the high prevalence of panic disorder in COPD and the high individual and social costs when panic disorder develops.

\section{STATEMENT OF INTEREST}

None declared.

\section{ACKNOWLEDGEMENTS}

The authors would like to thank the staff from the Department of Respiratory Medicine, Prince of Wales Hospital, Sydney, Australia, for their assistance with the administration of the study.

\section{REFERENCES}

1 Halbert R, Natoli J, Gano A, et al. Global burden of COPD: systematic review and meta-analysis. Eur Respir J 2006; 28: 523-532.

2 American Psychiatric Association. Diagnostic and Statistical Manual of Mental Disorders, 4th Edn. Washington DC, American Psychiatric Association, 1994; pp. 430-441.

3 Smoller J, Pollack M, Otto M, et al. Panic anxiety, dyspnea, and respiratory disease. Am J Respir Crit Care Med 1996; 154: 6-7.

4 Clark D. A cognitive approach to panic. Behav Res Ther 1986; 24: 461-470.

5 Oga T, Nishimura K, Tsukino M, et al. Longitudinal deteriorations in patient reported outcomes in patients with COPD. Respir Med 2007; 101: 146-153.

6 Katon W, Lin E, Kroenke K. The association of depression and anxiety with medical symptom burden in patients with chronic medical illness. Gen Hosp Psychiatry 2007; 29: 47-55.

7 Mitte K. A meta-analysis of the efficacy of psycho- and pharmacotherapy in panic disorder with and without agoraphobia. J Affect Disord 2005; 88: 27-45.

8 Wetherell J, Lenze E, Stanley M. Evidence-based treatment of geriatric anxiety disorders. Psychiatr Clin North Am 2005; 28: 871-896.

9 Hofmann S, Meuret A, Rosenfield D, et al. Preliminary evidence for cognitive mediation during cognitive-behavioral therapy of panic disorder. J Consult Clin Psychol 2007; 75: 374-379.

10 Salkovskis P, Hackmann A, Wells A, et al. Belief disconfirmation versus habituation approaches to situational exposure in panic disorder with agoraphobia: a pilot study. Behav Res Ther 2007; 45: 877-885.

11 Lisansky D, Clough D. A cognitive-behavioral self-help educational program for patients with COPD: a pilot study. Eur Respir J 1996; 10: 97-101.

12 Eiser N, West C, Evans S, et al. Effects of psychotherapy in moderately severe COPD: a pilot study. Psychother Psychosom 1997; 10: 581-584.

13 Emery C, Schein R, Hauck E, et al. Psychological and cognitive outcomes of a randomised trial of exercise among patients with chronic obstructive pulmonary disease. Health Psychol 1998; 17: 232-240.

14 Kunik M, Braun U, Stanley M, et al. One session cognitive behavioural therapy for elderly patients with chronic obstructive pulmonary disease. Psychol Med 2001; 31: 717-723.

15 De Godoy D, de Godoy R. A randomized controlled trial of the effect of psychotherapy on anxiety and depression in chronic obstructive pulmonary disease. Arch Phys Med Rehabil 2003; 84: 1154-1157.

16 Story $\mathrm{T}$, Zucker B, Craske M. Secondary prevention of anxiety disorders. In: Dozois D, Dobson K, eds. The Prevention of Anxiety and Depression: Theory, Research and Practice. Washington DC, American Psychological Association, 2004; pp. 131-160.

17 Kunik M, Veazey C, Cully J, et al. Group education and cognitive behavioral therapy group treatment for clinically significant symptoms of depression and anxiety in COPD patients: a randomized controlled trial. Psychol Med 2007; 9: 1-12.

18 Pauwels R, Buist A, Calverley P, et al. GOLD Scientific Committee. Global strategy for the diagnosis, management, and prevention of chronic obstructive pulmonary disease. NHLBI/WHO Global Initiative for Chronic Obstructive Lung Disease (GOLD) Workshop summary. Am J Respir Crit Care Med 2001; 163: 1256-1276.

19 Coventry P. Does pulmonary rehabilitation reduce anxiety and depression in chronic obstructive pulmonary disease? Curr Opin Pulm Med 2009; 15: 143-149.

20 Livermore N, Sharpe L, McKenzie D. Cognitive behaviour therapy for panic disorder in chronic obstructive pulmonary disease: two case studies. Behav Cogn Psychother 2008; 36: 625-630.

21 Brown T, DiNardo P, Barlow D. The Anxiety Disorders Interview Schedule for DSM-IV. Albany, Graywind Publications, 1994; pp. 1-100. 
22 Zigmond A, Snaith R. The Hospital Anxiety and Depression Scale. Acta Psychiatr Scand 1983; 67: 361-370.

23 Sutton K, Cooper M, Pimm J, et al. Anxiety in chronic obstructive pulmonary disease: the role of illness specific catastrophic thoughts. Cognit Ther Res 1999; 23: 573-585.

24 Jones P, Quirk F, Baveystock C. The St. George's Respiratory Questionnaire. Respir Med 1991; 85: Suppl. B, 25-31.

25 Gardenswartz C, Craske M. Prevention of panic disorder. Behav Ther 2001; 32: 725-737.

26 Dobson K, Dozois D. The prevention of anxiety and depression: promises and prospects. In: Dozois D, Dobson K, eds. The Prevention of Anxiety and Depression: Theory, Research and Practice. Washington DC, American Psychological Association, 2004; pp. 283-295.

27 Carrieri-Kohlman V, Murray Gormley J. Coping strategies for dyspnea. In: Mahler D, ed. Dyspnea. New York, NY, Marcel Dekker, 1998; pp. 287-320.

28 Gallagher-Thompson D, Thompson L. Applying cognitive-behavioral therapy to the psychological problems of later life. In: Zarit S, Knight B, eds. A Guide to Psychotherapy and Aging: Effective Clinical Interventions in a Life-Stage Context. Washington DC, American Psychological Association, 1996; pp. 61-82.
29 Bourbeau J, van der Palen J. Promoting effective self-management programmes to improve COPD. Eur Respir J 2009; 33: 461-463.

30 Zvolensky M, Schmidt N, Bernstein A, et al. Risk-factor research and prevention programs for anxiety disorders: a translational research framework. Behav Res Ther 2006; 44: 1219-1239.

31 Pollack M, Smoller J. The longitudinal course and outcome of panic disorder. Psychiatr Clin North Am 1995; 18: 785-801.

32 Cully J, Graham D, Stanley M, et al. Depressed and anxious COPD patients: predictors of psychotherapy engagement from a clinical trial. J Clin Psychol Med Settings 2007; 14: 160-164.

33 Coventry P, Hind D. Comprehensive pulmonary rehabilitation for anxiety and depression in adults with chronic obstructive pulmonary disease: systematic review and meta-analysis. J Psychosom Res 2007; 63: 551-565.

34 Maurer J, Rebbapragada V, Borson S, et al. Anxiety and depression in COPD: current understanding, unanswered questions, and research needs. Chest 2008; 134: Suppl. 1, 43-56.

35 Australian Lung Foundation. Economic Case statement: Chronic Obstructive Pulmonary Disease. Lutwyche, Australian Lung Foundation, 2002; pp. 12-17.

36 Ries A. Pulmonary rehabilitation and COPD. Semin Respir Crit Care Med 2005; 26: 133-141. 